\title{
Mittelalterliche bis spätmittelalterliche Dünenbildung in Cornwall und deren Beziehung zum „Little Ice Age” in Mitteleuropa
}

\author{
Helmut Siebertz*) \\ Holocene, stratigraphy, blown sand deposits, fossil soils, high- and late mediaeval climate, \\ atmospherical circulation, Little Ice Age, Cornwall, North-West-Europe
}

\begin{abstract}
Kurzfassung: An der Nordwestküste Cornwalls (Abb. 1) liegen Jungdünen in stratigraphischer Abfolge zu älteren Flugdecksanden. In Gwithian (Abb. 2) konnten zwei fossile Böden (G 2-Horizont mit $688 \pm 61$ B.P., cal $1335 \pm 51$ A.D.; G 5-Horizont mit $404 \pm 68$ B.P., cal 1527 \pm 76 A.D.) bestimmt werden, deren Sedimentablagerung sich gut in die hoch- bis spätmittelalterliche Klimasitutation des nordwesteuropäischen Raumes einfügt. Mit der im 16. Jahrhundert einsetzenden „Kleinen Eiszeit" kommt es nur noch zur geringen Sedimentanwehung, die aufgrund der Klimaverschlechterung schwach ausgebildete Bodenhorizonte hervorbringt (Abb. 2). Eine erneute Dünensandbildung findet dann erst wieder in der Neuzeit statt.
\end{abstract}

\section{[Blown sand deposits of high- and late-mediaeval age at Cornwall and the connection to the "Little Ice Age" in the middle European area]}

\begin{abstract}
At the north-west coast of Cornwall (Fig. 1) younger blown sand (dune sand) lies in stratigraphical order to older blown sand deposits. Two fossil soils are dated by the ${ }^{14} \mathrm{C}$-method at Gwithian. The older lower soil is aged at $688 \pm 61$ B.P. (cal $1335 \pm 51$ A.D.), and the younger upper soil is dated at $404 \pm 68$ B.P. (cal $1527 \pm$ 76 A.D.). The most of the blown sand deposits of that profile are of high- and late-mediaeval age (Fig. 2); the deposits of dune sand at that period are in the right manner to the climatic situation in North-West Europe. At the beginning of the "Little Ice Age" in the 16. century the drift of blown sand is almost interrupted and the bad climatic conditions have led to the production of only small humous soil mineralisation (Fig. 2). After that period the blown sand deposits are reactivated (recent time).
\end{abstract}

\section{Fragestellung}

Der Südwesten von England bildet eine Plateaufläche, die überwiegend aus devonischen Sandsteinen und Schiefern aufgebaut wird und von einer Reihe von Granitplutonen (Batholithen)

\footnotetext{
*) Anschrift des Verfassers: Dipl.-Geogr. (phys.) Dr. rer. nat H. Siebertz, Alte Schulstraße 16, 53229 Bonn-Bechlinghoven
}

durchsetzt ist (Abb. 1). Nach Leuze (1961) sind dem armorikanischen Faltenrumpf eine Reihe alter Landoberflächen und mariner Abrasionsflächen aufgeprägt, die sich - ohne Rücksicht auf die Gesteinsunterschiede - über weite Teile des Landes hinwegziehen. Neuere Untersuchungen von Couqe-Delhuille (1991) modifizieren dies. Entsprechend der geologisch-geomorphologischen Struktur ist der Küstenraum überwiegend von Kliff- und Steilküsten geprägt, die lokal von ausgeprägten Buchten unterbrochen sind. In diesen Buchten sind durch litorale Prozesse teilweise breite Sandstrände entstanden, die als Liefergebiete für eine Reihe von äolischen Sedimenten dienen (Siebertz 1994). Nach Catt \& Staines (1982) sowie Roberts (1985) befinden sich zwischen dem Pendennis Point (Falmouth) sowie dem Lizard Point Lößablagerungen, die in ihrer Genese an das verwitterte unterlagernde Gestein gebunden sind und in ihrer Sedimentstruktur wahrscheinlich (nicht unmittelbar) mit den mitteleuropäischen Lössen vergleichbar sind.

Die Süd- als auch die Nordwestküste werden entlang der Buchten von Flugdecksanden und Dünen begleitet, wobei die älteren Flugsedimente auf den Kliffs lagern, die jüngeren Dünen sich aber meist in den Hängen der Buchten befinden und sich bis auf die Hochfläche hinaufziehen. Dort liegen häufig die älteren Flugdecksande im Liegenden und die jüngeren Dünensande im Hangenden. Die Jungdünen zeigen nach BARTON (1964) eine große Mächtigkeit; sie haben mittlerweile mehrere alte Kirchen begraben (z. B. Saint Pirans Church in den Penhale sands von Perranporth).

Über das Alter der Flugsedimente liegen bisher kaum Ergebnisse vor. So konnten von SIEBERTz (1994) Flugdecksande in der Bucht von Saint Ives auf dem Black Cliff datiert werden, die genetisch mit dem Pleniglazial der Weichsel-Kaltzeit beginnen und im Mittelholozän enden. Neuere Untersuchungen an jüngeren Dünensanden in Gwithi- 

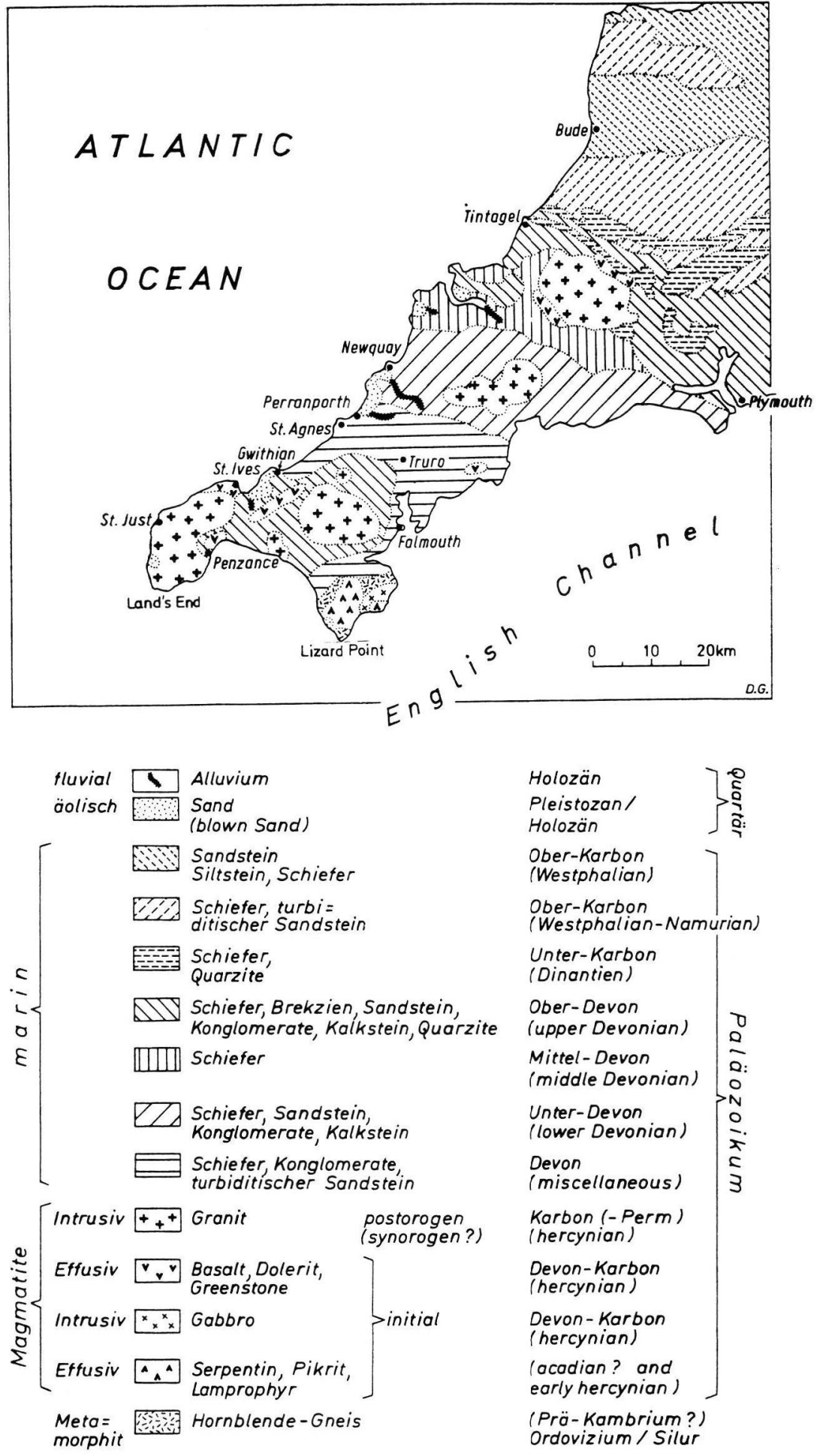

$\left.\begin{array}{l}\text { Holozän } \\ \text { Pleistozan/ } \\ \text { Holozän } \\ \text { Ober-Karbon } \\ \text { (Westphalian) } \\ \text { Ober-Karbon } \\ \text { (Westphalian-Namurian) } \\ \text { Unter-Karbon } \\ \text { (Dinantien) } \\ \text { Ober-Devon } \\ \text { (upper Devonian) } \\ \text { Mittel-Devon } \\ \text { (middle Devonian) } \\ \text { Unter-Devon } \\ \text { (lower Devonian) } \\ \text { Devon } \\ \text { (miscellaneous) } \\ \text { Karbon (-Perm) } \\ \text { (hercynian) } \\ \text { Devon-Karbon } \\ \text { (hercynian) } \\ \text { Devon-Karbon } \\ \text { (hercynian) } \\ \text { (acadian? and } \\ \text { earlyhercynian) } \\ \text { (Prä-Kambrium?) } \\ \text { Ordovizium / Silur }\end{array}\right]$

Abb. 1: Geologische Skizze von Cornwall (zusammengestellt nach BarTon 1964, Bristow 1996, Brown 1984, EDMONDs et al. 1985).

Fig. 1: Geological map of Cornwall (compiled after BARTON 1964, Bristow 1996, Brown 1984, Edmonds et al. 1985). 
an (in unmittelbarer Nähe von Hayle; Abb. 1) zeigen, daß die Jungdünen in historischer Zeit noch aktiv und überwiegend mittelalterlich (prä Little Ice Age) angelegt sind (Abb. 2). Danach beginnen die Sedimentationsraten (in diesem Raum) schwächer zu werden, so daß auch innerhalb der Wanderdünen eine gewisse Stabilität eintritt.

Das datierte Profil von Gwithian wird stratigraphisch beschrieben und mit ähnlichen Ablagerungen im nordwesteuropäischen Raum diskutiert. Ferner soll der Versuch unternommen werden, die jungen Flugsedimente klimatisch mit der „Kleinen Eiszeit" (Little Ice Age) in Verbindung zu setzen.

\section{Profilaufbau und Profilbeschreibung}

Das Sedimentprofil befindet sich am Ortsausgang von Gwithian in Richtung Portreath; es liegt im Scharnier zwischen Strand und felsigem Anstehenden, dessen Höhen rasch bis auf $30 \mathrm{~m}$ ansteigen. Die Höhenlage der Dünensande kann entsprechend der topographischen Karte 1:25.000 Saint Ives Sheet SW54 mit etwa 5-8 m angenommen werden. Das Profil befindet sich an der Nahtstelle zweier Sedimentationsräume; der geologische Untergrund wird entsprechend der geologischen Karte von BROWN (1984) von marinem Oberdevon bzw. gemischten klastischen Sedimenten des Devons aufgebaut (Abb. 1), deren Verwitterungsprodukte in der Saint Ives Bay als litorale Ablagerungen vorzufinden sind und als Liefergebiete für die Flugsanddecken sowie Dünensande dienen. Sie sind in diesem Raum meistens von Headablagerungen, einer periglazialen Sedimentfazies von abgetragenen und umgelagerten Materialien unterschiedlicher Zusammensetzung, unterlagert. Dem geologisch-geomorphologischen Untergrund folgt eine bis zu $2 \mathrm{~m}$ mächtige nicht zu differenzierende Dünensandablagerung. Der Dünensand ist meist hell, lokal leicht ockerfarbig. Die schwachen Oxidationen deuten auf einen geringen Anteil an Ferralliten hin (Abb. 2). Die Sedimentation zeigt söhlige Ablagerungen und Kreuzschichtungen. Auch Schnecken kommen darin vor.

Diesem Sedimentkomplex folgt eine sehr differenzierte, knapp $40 \mathrm{~cm}$ mächtige Dünensandbildung, die von zahlreichen mehr oder weniger stark ausgeprägten humosen Horizonten (zersetzte mineralisierte Oh-Horizonte) durchsetzt ist. In den meisten Fällen finden sich in den Sedimentabschnitten nur Spuren von humosen Mineralstoffen. Ihnen folgt im Hangenden ein Horizont mit umgelagerten Kiesen sowie eine graue

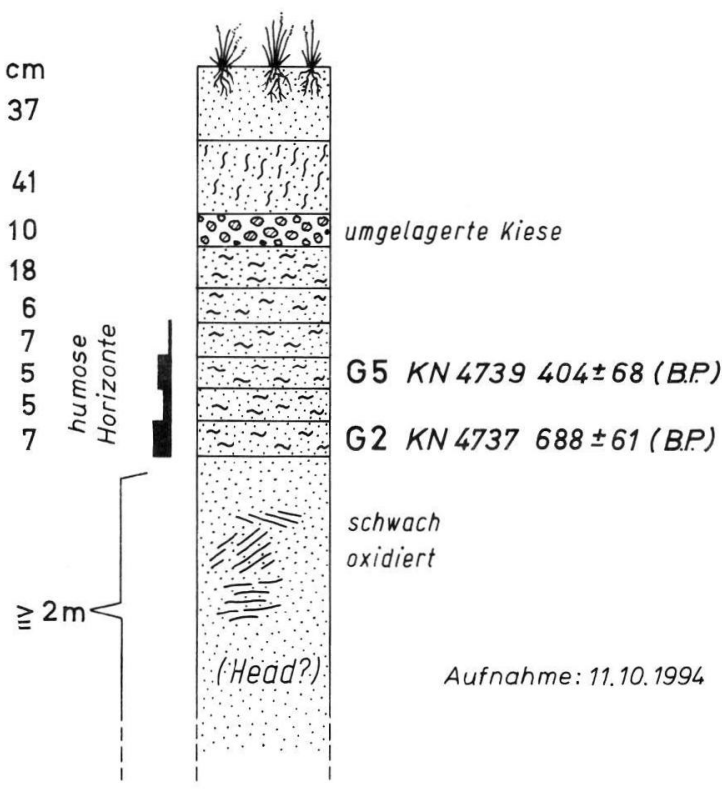

Abb. 2: Chronostratigraphisches jüngeres Dünensandprofil von Gwithian.

Fig. 2: Chronostratigraphical profile of younger blown sand deposits at Gwithian.

Sandschicht, welche durch die Auswaschungsprozesse aus dem rezenten Boden verursacht worden ist. Die Korngrößenzusammensetzung (Tab. 1) entspricht den Werten, wie sie bereits von SieberTz (1994) für diverse Dünensande in diesem Raum ermittelt wurden.

\section{Radiometrische Altersdatierung}

Die radiometrischen Altersdatierungen der fossilen Bodenhorizonte in den Dünen von Gwithian sind durchweg sehr jung. Sie umfassen ein konventionelles Alter, welches Bodenbildungen aus dem 14. und 16. Jahrhundert umfaßt. So liegt das ${ }^{14} \mathrm{C}$-Alter des fossilen $\mathrm{G} 2$-Horizontes bei $688 \pm 61$ B.P.; dies entspricht einem kalibrierten Datum von $1335 \pm 51$ A.D. Der jüngere $G 5$-Horizont trägt ein ${ }^{14} \mathrm{C}$-Alter von $404 \pm 68$ B.P. Jahren, welches kalibriert einem konventionellen Alter von $1527 \pm 76$ A.D. vergleichbar ist.

Nach Weninger (1995) werden die angeführten konventionellen ${ }^{14} \mathrm{C}$-Alter mit der Libby-Halbwertszeit von 5568 Jahren berechnet, wobei die physikalische Einheit B.P. (Before Present) 0 B.P. $=1950$ A.D. entspricht. Die Daten sind bezogen auf den NBS-Oxalsäure-Rezentstandard und damit der internationalen Radiokohlenstoff-Zeitskala angepaßt.

Für eine archäologische Auswertung sind nach WenINGER (1995) in aller Regel die kalibrierten 
Tab. 1: Strand- und Dünensande von der Nordwestküste Cornwalls.

Tab. 1: Grain-size distribution of beach and blown sand deposits at the north-west coast of Cornwall.

\begin{tabular}{lccc|c|ccc|cc}
\hline \multicolumn{3}{c|}{ Strand Hayle } & & $\begin{array}{c}\text { Jungdünen } \\
\text { Hayle }\end{array}$ & \multicolumn{2}{c|}{$\begin{array}{c}\text { Strand } \\
\text { Perranporth }\end{array}$} & \multicolumn{2}{|c}{$\begin{array}{c}\text { Jungdünen } \\
\text { Perranporth }\end{array}$} \\
\hline $\mathrm{mm}$ & & & & & & & & & \\
$2-1$ & 1,5 & 5,9 & 2,8 & 1,0 & - & - & - & - & - \\
$1-0,63$ & 6,5 & 18,8 & 11,3 & 8,0 & - & 0,2 & - & 0,1 & - \\
$0,63-0,2$ & 87,0 & 71,8 & 81,0 & 87,0 & 81,8 & 89,7 & 91,4 & 81,4 & 74,4 \\
$0,2-0,1$ & 2,2 & 0,8 & 1,7 & 2,9 & 14,2 & 7,9 & 6,0 & 16,7 & 23,6 \\
$0,1-0,063$ & - & - & - & 0,1 & 0,1 & - & 0,1 & 0,1 & 0,1 \\
$0,060-0,020$ & - & 0,1 & - & - & 0,4 & 0,5 & - & - & 0,4 \\
$0,020-0,006$ & 0,4 & - & 0,1 & - & - & - & 0,2 & - & - \\
$0,006-0,002$ & - & - & - & 0,1 & 0,8 & - & 0,4 & 0,6 & 0,5 \\
$<0,002$ & 2,4 & 2,6 & 3,1 & 0,9 & 2,7 & 1,7 & 1,9 & 1,1 & 1,0 \\
\hline
\end{tabular}

(dendrochronologisch geeichten) ${ }^{14} \mathrm{C}-\mathrm{Daten} \mathrm{zu}$ benutzen. Diese kalibrierten ${ }^{14} \mathrm{C}$-Daten (cal AD, cal BC) sind direkt mit historischen Daten vergleichbar, so auch mit den Daten der Dendrochronologie sowie der Thermolumineszenz. Das „wahre" Probenalter liegt mit einer statistischen Sicherheit von $68 \%$ innerhalb des berechneten Zeitintervalls der Kalenderzeitskala.

Zwischen dem G 2- und G 5-Horizont liegt ein im Profil nicht vermerkter, umgelagerter Horizont ( $G$ 3). Dieser Horizont ist aufgrund der Durchlässigkeit des Dünensandes und der Vermischung von fremden Substanzen aus den höher liegenden Schichten verunreinigt und für eine genaue chronostratigraphische Ausdeutung von geringer Bedeutung. Die Verunreinigungen im Profil jedoch zeigen, wie dies auch aus den umgelagerten Kiesen im oberen Profilteil hervorgeht, daß neben einer kontinuierlichen Ablagerung über Jahrhunderte hinweg auch Umlagerungen und Verwehungen bei den jüngeren Dünensanden stattgefunden haben.

\section{Das Profil von „Gwithian” und seine Stellung zum „Little Ice Age” in Mitteleuropa}

Die beiden Profilhorizonte G 2 und G 5 fallen historisch in eine Zeit, die synoptisch nur durch Beschreibungen, Briefe und Mitteilungen überliefert ist, so daß aufgrund emotionaler Schilderungen zuweilen die Genauigkeit in Frage gestellt ist; die Daten der Profile sprechen jedoch zunächst einmal für sich. Erste regelmäßige synoptische Aufzeichnungen finden im 17. Jahrhundert statt und nehmen dann bis in die heutige Zeit an Kontinuität und Genauigkeit zu.

Das Hochmittelalter (etwa 1000-1200 n.Chr.) wird nach FlOHN $(1967,1985)$ als das mittelalterliche
Klimaoptimum angesehen, in dem der Sommer sowie der Herbst im Durchschnitt höhere Temperaturen und selten Maifröste aufwiesen, als dies heute der Fall ist. Ein entsprechendes ,little Optimum" wird für England von LAMB (1966) für den Zeitraum um 1000 bis 1300 A.D. erwähnt. Nach Flohn (1957) lagen die Temperaturen im Sommer sowie im Winter ebenso hoch wie in der Zeit maximaler rezenter Erwärmung, vielleicht sogar um $0,5-1^{\circ} \mathrm{C}$ höher nach indirekten Klimazeugen. In diese Warmzeit fällt die Rottnest-Transgression (FLOHN 1967), die mit einem deutlichen Meeresanstieg verbunden ist. Diese Zeit ist auch geprägt durch das geringe Vorkommen von Stürmen; die niedrige Zyklonenhäufigkeit auf dem Atlantik und den Rückzug des Meereises, verbunden mit einem Fehlen von Treibeis in den grönländischen Gewässern bis $70^{\circ} \mathrm{N}$ hinauf. Sie hängen nach FlOHN (1967) ursächlich zusammen.

Das Klimaoptimum wird - soweit die sedimentologischen Voraussetzungen dafür vorliegen auch durch rezente Bodenbildungen nachgezeichnet. So lassen sich in dem hochmittelalterlichen Zeitraum diverse Böden - meistens Plaggenesche - nachweisen, die nicht nur die günstigen Klimabedingungen nachzeichnen, sondern auch den Hinweis für eine vorausgehende Sedimentanwehung aufzeigen. Die Bildung der Plaggenesche wird nach MückenHausen et al. (1968) hauptsächlich auf die Zeit von 800-1200 Jahren datiert. So werden nach CASTEL et al. (1989) die Ausdehnungen der Plaggenböden im nordwestlichen Europa zwischen 750 und 1200 A.D. besonders hervorgehoben. Die Voraussetzung dafür ist die verstärkte äolische Umlagerung von terrestrischen Sedimenten; in den meisten Fällen handelt es sich um spätglaziale (jüngere) Flugsanddecken, die nach CASTEL et al. (1989) seit dem Beginn des Neolithikums eine weitverbreitete Akku- 
mulation von Binnendünen oder Flugsanddecken zur Folge hatten.

Altersdatierungen liegen für das Klimaoptimum überwiegend aus dem nordwestlichen europäischen Raum vor, so für die Niederlande von CASTel et al. (1989), KLijn (1990), Jelgersma et al. (1970) und DeJong (1984). Für England sind entsprechende Werte von Tooley (1990) sowie von WiLSON (1990) für Nordirland zusammengestellt worden. Die meisten Untersuchungen decken allerdings nur das Früh-Neolithikum und ältere holozäne Sandanwehungen mit entsprechenden Bodenbildungen $\mathrm{ab}$.

Nach dem Klimaoptimum setzt nach FloHN (1967) etwa ab 1164, verstärkt aber um 1200 eine Zunahme von Stürmen und Sturmfluten ein, die zwischen 1250-1400 häufige Niederschläge und Stürme in England sowie an der Nordseeküste hervorrufen (FlOHN 1957). Die Sturmflut von 1250/1251 führt zur Bildung der Zuider See in Holland. Diese kalte Periode zwischen 1200 und $1500 \mathrm{n}$. Chr. geht mit einer marinen Regression (Paria-Regression) einher, der in Süddeutschland sowie in der Schweiz ein Höhepunkt der Klimagunst entgegensteht (etwa 1280-1380). Hier gab es Witterungsextreme im Sommer sowie im Winter. Es wurde allerdings nicht die Klimagunst des Hochmittelalters erreicht.

Auch nach LAmB (1966) ist die Zeit des späten Mittelalters von Sturmfluten gekennzeichnet (1240-1253, 1267-1292), wobei von 1300 bis 1550 im gesamten englischen Raum starke Westwinde herrschten. In dieser Zeit werden die Basissande des Profils akkumuliert, in denen sich vor dem Kälteschock ab Mitte des 16. Jahrhunderts die beiden mineralisierten Horizonte G 2 und G 5 (Abb. 2) bilden können. Eine ähnliche zeitliche Entwicklung zeigte sich bei der Ablagerung der Wisseler Dünen im unteren Niederrheingebiet, deren Bildung nach Höppner \& STEEger (1936) im späten Mittelalter einsetzte.

Der Klimabesserung in der ersten Hälfte des 16. Jahrhunderts, bestätigt durch den G 5-Horizont in Abb. 2, folgt nach FLOHN (1957) eine markante Klimaverschlechterung, wobei die Jahresmitteltemperatur um schätzungsweise $1,5^{\circ} \mathrm{C}$ absank. Diese kalte Klimaperiode der Zeit von 1560 bis fast 1700 wird von den britischen Klimahistorikern (nach Gletschervorstößen in den Alpen) „etwas übertreibend als „Kleine Eiszeit” (zit. nach FlOHN 1957,1967) bezeichnet mit sinkenden Wintertemperaturen um $1,5-2^{\circ} \mathrm{C}$; dies hatte zur Folge, daß die Eisverhältnisse um Island ab 1540 , besonders ab 1600, katastrophal zunahmen. Diese Zeit ging mit einer relativen Trockenheit in England und Belgien einher (FLOHN 1957).

Über den Beginn und die Dauer des „Little Ice Age" sind die Autoren unterschiedlicher Auffassung. So wird von Flohn (1985) der Zeitraum von 1550 bis 1850 angegeben, nach GribBIN \& LAMB (1978) die Zeit von 1430 bis 1850 und nach LAMB (1982) zwischen 1550 und 1900. Der Höhepunkt der "Kleinen Eiszeit" wird von PFISTER (1982) mit den Jahren 1688-1701 benannt, wobei als Ursache eine temporäre Abschwächung der Sonneneinstrahlung vermutet wird. Trotz der Abkühlung der Jahreszeiten ab 1525, die mit verstärkten Niederschlägen in den Sommern einhergingen, gab es auch temperaturmäßig Höhepunkte, wie der um 1540 in Mitteleuropa auftretende 10 Monate anhaltende Subtropengürtel zeigt (PFISTER 1982). Innerhalb der „Kleinen Eiszeit“-Periode gab es nach FloHn (1985) aber immer wieder mildere Abschnitte, sogar sehr warme Einzeljahre; dabei war die Variabilität der Temperaturen deutlich größer als heute.

Dies drückt sich auch in den humosen Horizonten oberhalb des G 5-Abschnittes aus. Sie sind qualitativ nur mäßig ausgebildet und liegen dicht zusammen, so daß eine klare Trennung der Horizonte nicht mehr möglich ist (Abb. 2). Während der Kälteperiode hielt sich die Sedimentanwehung allerdings deutlich in Grenzen. Der G 5-Horizont findet daher auch relativ wenig parallele Datierungen im Küstenraum Englands sowie im nordwestlichen Küstengebiet Mitteleuropas.

So liegen lediglich für das belgische sowie das niederländische Küstengebiet fossile Bodenhorizonte vor, die eine Bodenbildung im 16. Jahrhundert beschreiben und eine Überleitung zum "Little Ice Age" bilden. Ausgiebige Vorkommen beschreibt CASTEL (1991), die den Zeitraum von etwa 390-435 a B.P. umschreiben (u.a. $400 \pm 90$ B.P.) und selbst das Binnenland beinhalten. DE JONG (1984) weist bei Untersuchungen der niederländischen friesischen Inseln im sandigen Torf ein Alter von $430 \pm 45$ B.P. nach, der etwa der Bildung des G 5-Horizontes in Cornwall entspricht. Auch für die belgische Küste liegt eine beinahe gleichwertige Datierung von DE CEunYNCK (1985) vor $(430 \pm 75$ B.P.). Von dem Autor werden jedoch verschiedene jahreszeitliche Perioden beschrieben, die im 14. und 15. Jahrhundert charakteristisch sind für die Bildung von fossilen Böden in Flugsanden und Dünen (z. B. 1280-1410 A.D., 1320-1420 A.D., 1400-1515 A.D.). Die meisten anderen vorliegenden Befunde in der Literatur betreffen überwiegend die Zeit um Christi Geburt 
oder sind älter, so daß Altersdatierungen - wie die von Gwithian - (bisher) meistens nur lokale Einzelfälle bleiben.

\section{Die Zirkulationsverhältnisse in den höheren geographischen Breiten während des „Little Ice Age“}

Die atmosphärischen Zirkulationsmechanismen sind abhängig von den Temperaturgegensätzen zwischen den Polen und dem Äquator; sie initiieren einen Druckgradienten, der die Bewegung der Luftmassen auslöst. Der Luftmassenaustausch findet in den mittleren geographischen Breiten der Nordhalbkugel zwischen $35^{\circ}$ und $65^{\circ}$ statt; dieser Raum wird als „planetarische Frontalzone” bezeichnet, wo der Wind in der Höhenströmung etwa im 500 mb-Niveau mit einer mehr oder weniger starken West-Ost-Strömung über Mitteleuropa weht. Diese Zirkulation ist die Modellvorstellung als Normalfall, welcher jedoch im Laufe der erdgeschichtlichen Entwicklung in seiner Regelhaftigkeit des öfteren unterbrochen wurde und für „zirkulationsbedingte Ausreißer“ (z. B. Eiszeiten) gesorgt hat.

Der Auslöser für das Verhalten der Luftstromveränderungen und damit verbunden die Auswirkungen extremer Klimate im Sommer und Winter, wie dies im „Little Ice Age“ der Fall war, sind erklärungsbedürftig. So geht FloHN $(1967,1985)$ von einer verstärkten Meridionalzirkulation aus, die nach GriBbin \& LAMB (1978) durch einen hohen meridionalen Temperaturgradienten in der kalten Phase zwischen $40^{\circ}$ und $60^{\circ} \mathrm{N}$ verursacht worden sein muß. Eine hohe Sonnenaktivität in der zweiten Jahreshälfte führt nach FloHN (1967) dazu, daß sich diese im Winter in einer Steigerung der Zonalzirkulation auswirkt. Beim Ausbleiben derselben tritt in den Wintern verstärkt eine Meridionalzirkulation hervor; ist das Druck-ÜberschußGebiet nach Westen verlagert (Atlantik zwischen Norwegen, Island, Färöer), so wird das Auftreten milder West- und Süd-West-Wetterlagen unterbunden.

Der Antriebsmechanismus für die Schwankungen bei den Sonnenstrahlen werden nach FLOHN (1967) sowie Lamb (1979) mit einer Verstärkung der Vulkanausbrüche in Verbindung gebracht. Die Erdgeschichte zeigt, daß die großen Vereisungen sich nach orogenen Vorgängen mit häufig weltweitem Vulkanismus einstellten, so daß hypothetisch hier ein kausaler Zusammenhang vorliegen könnte. So ist nicht verwunderlich, daß die höhere Atmosphäre durch Vulkanstaub die solaren Aktivitäten hemmt. Aber selbst Zeiten extre- mer Abkühlung können sommerliche Hitzeperioden gegenüberstehen, wie die „Great Plague in 1665 and the Fire of London in 1666" deutlich zeigen (s. LAMB 1979).

Die extreme Abkühlung führte in Mitteleuropa zu einer verstärkten Meridionalzirkulation mit „blocking action” in der Westwinddrift; diese Zirkulationsanomalie hatte nach FloHN (1967) zur Folge, daß eine Verstärkung des quasistationären Höhentroges über Osteuropa bestand und sich zugleich eine Verlagerung desselben nach Westen (etwa $95^{\circ} \mathrm{W}, 10$ bis $15^{\circ} \mathrm{E}$ ) auswirkte, wobei eine Verstärkung der Hochdruckrücken über der Küste Westeuropas und blockierende Hochdruckgebiete im Winter über Skandinavien, im Sommer über dem Nordmeer und den Britischen Inseln stattfand. Dadurch wurde im ganzen amerikanisch-europäischen Sektor die Hauptfrontalzone äquatorwärts abgedrängt, so daß meridionale Zirkulationstypen (low index Zirkulation) überwogen. Solche Zirkulationen rufen häufig Ostwetterlagen hervor, die nach LENkE (1968) in den Wintermonaten ein im Vergleich zur Neuzeit zu kaltes und schneereiches Klima geben; die Folge war, daß die Sommermonate bei besonders häufigen West-, Nordwest- und Nordwinden im Mittel durch größere Unbeständigkeit und offenbar auch zu kühle Witterung gekennzeichnet waren, was in dem Zeitraum von 1600 bis $1650 \mathrm{zu}$ nachgewiesenen Gletschervorstößen führte. Die Drehung der vorherrschenden Windrichtung von heute SW auf SE bei Ostwetterlagen ist nach FLOHN (1985) ein eindeutiger Hinweis auf eine Zirkulationsanomalie, wobei die Abkühlung und die häufig blockierenden Hochs im Raume der Nordsee und Skandinaviens als ein Anzeichen für die Vorherrschaft von Wetterlagen zu deuten sind, die zu Gletscherwachstum führen.

FLOHN (1967) ist der Auffassung, daß in warmen Perioden (postglaziales Wärmeoptimum, frühmittelalterliche und rezente Wärmezeit) die Westdrift auf dem Atlantik um 3 bis 5 Breitengrade nach Norden verschoben war, während in kalten Perioden (z. B. Höhepunkt der letzten Eiszeit und „Kleine Eiszeit”) die Zirkulationssysteme unter Abschwächung der Westdrift südwärts wanderten. Dabei verlagerten sich die Höhentröge auch westwärts, was zu einer Verstärkung des Meridionalaustausches mit häufigen Kaltlufteinbrüchen (nach Flohn 1985 Bildung extremer Wetterlagen mit Kaltlufttropfen) bis in das Mittelmeer hinein führte, ausgelöst in der „Kleinen Eiszeit” durch die ab 1500 gesteigerte vulkanische Aktivität. 
Die Zirkulationsverhältnisse im "Little Ice Age" zeigen, daß diese sehr ähnlich waren mit denen der letzten Kaltzeit (Weichsel-Glazial). Zu dieser Diskussion hat SIEBERTz (1988) Stellung genommen. Die Luftdruck- und Windverhältnisse zeigen, daß das Hochmittelalter und die nachfolgende Phase die Verlagerung von Flugsanden sowie die Bildung von Dünen plausibel machen, so daß bei einer Stabilisation und klimatischen Besserung sich relativ schnell humose Horizonte bilden konnten, wie sie in Abbildung 2 zum Ausdruck kommen; durch solche Zeitmarken werden im mittel- und nordwesteuropäischen Raum landschaftsgenetische Vorgänge dokumentiert, die für die Rekonstruktion vergangener Klimate (Paläoklimatologie) zweifellos von großer Bedeutung und Nutzen sind.

\section{Ergebnis der Untersuchungen}

Ältere Flugdecksande und jüngere Dünensande lagern auf den Kliffs und in den Hängen der Buchten oft stratigraphisch in enger Beziehung zueinander (vgl. SiEBERTz 1994). Junge Dünensande, die durch fossile mineralisierte Horizonte gegliedert sind, lassen sich im nordwesteuropäischen Raum relativ selten nachweisen. Dies hängt mit den Ablagerungsbedingungen in historischer Zeit und der in Ruhephasen der Sedimentation sich einstellenden humosen Mineralisierung zusammen.

So konnten an der Nordwestküste Cornwalls (Abb. 1) zwei fossile Horizonte in Dünensanden mit der ${ }^{14} \mathrm{C}$-Methode datiert werden (Abb. 2). Der ältere untere G 2-Horizont besitzt ein Alter von $688 \pm 61$ B.P. (1335 \pm 51 A.D.), der jüngere obere Horizont umfaßt den Zeitraum von $404 \pm 68$ B.P. (1527 \pm 76 A.D.). Sie fallen historisch in die Zeit des Hoch- und Spätmittelalters.

Die Basissande des Profils fallen nach LAMB (1966) in eine Zeit, die in England mit Sturmfluten und starken Westwinden einherging. Klimagünstige Zeitabschnitte ermöglichten eine humose Mineralisation mit der Bildung von Oh-Horizonten, so daß sich bis zum Beginn des „Little Ice Age” (Kleine Eiszeit) in der Mitte des 16. Jahrhunderts, die mit einer rapiden Klimaverschlechterung einsetzte, zumindest zwei datierungswürdige fossile Bodenhorizonte bilden konnten, die in Nordwesteuropa wenig Parallelen finden. Danach läßt nicht nur die Sandanwehung nach, sondern auch die Bildung von mineralisierten humosen Sedimentabschnitten im Profil (Abb. 2) - trotz lokal auftretender klimagünstiger Sommer.
Die Gründe für die Klimaverschlechterung werden in einem verstärkten Vulkanismus und damit verbunden einer Verminderung der auf der Erde auftreffenden Sonnenstrahlung gesehen. Zirkulationsbedingt hat sich damit eine „low index”Strömung eingestellt, die durch blocking action in der Höhenströmung der Westwindzone (etwa im $500 \mathrm{mb}$-Niveau) eine Meridionalzirkulation hervorrief, deren Höhentröge mit einer mehr oder weniger starken Nordströmung Kaltluft nach Süden verfrachteten, so daß sich nach FLOHN (1985) Kaltlufttropfenbildung mit entsprechender winterlicher Wetterlage einstellen konnte.

\section{Danksagung}

Herrn Dr. Bernhard Weninger vom Institut für Urund Frühgeschichte der Universität Köln danke ich für die ${ }^{14} \mathrm{C}$-Altersdatierung der fossilen Bodenhorizonte des Dünensandprofils von Gwithian (Cornwall).

\section{Schriftenverzeichnis}

BARTON, R.M. (1964): An introduction to the Geology of Cornwall, 1-168; Truro (Barton Ltd.).

Bristow, C.M. (1996): Cornwall's Geology and Scenery, 1-148; Saint Austell (Cornish Hillside Publ.).

Brown, G.M. [Dir.] (1984): British Geological Survey 1:50.000, Penzance, Sheet $351 \& 358$; Southampton.

CASTEL, I.I.Y. (1991): Late Holocene eolian drift sands in Drenthe (The Netherlands). - Nederlandse Geograf. Stud., 133: 1-156; Utrecht.

- Koster, E. \& Slotboom, R., (1989): Morphogenetic aspects and age of Late Holocene eolian drift sands in Northwest Europe. - Z. Geomorph., N.F., 33: 1-26, 10 Fig., 2 Phot., 2 Tab.; Berlin-Stuttgart.

CatT, J. A. \& Staines, S. J. (1982): Loess in Cornwall. Proc. of the Ussher Society, 5: 368-375, 3 Fig., 2 Tab.; Camborne.

Cenunynck, R. De (1985): The Evolution of the Coastal Dunes in the Western Belgian Coastal Plain. - Eiszeitalter u. Gegenwart, 35: 33-41, 3 Fig., 1 Tab; Hannover.

Couqe-Delhuile, B. (1991): The long term geomorphologic evolution of the English South-West massif (UK). - Z. Geomorph., N.F., 35: 65-84, 5 Fig., 1 Tab.; Berlin-Stuttgart.

DeJong, J. (1984): Age and Vegetational History of the Coastal Dunes in the Frisian Islands, The Netherlands. - Geol. en Mijnb., 63: 269-275, 4 Fig., 1 Tab.; Leidschendam.

Edmonds, E. A., McKfown, M. C. \& Williams, M. (1985): British Regional Geology, South-West England, 1138, 33 Fig., 12 Plates; London (Her Majesty's Stationery Office).

FloHN, H. (1957): Klimaschwankungen der letzten 1000 Jahre und ihre geophysikalischen Ursachen. - Ta- 
gungsber. u. wiss. Abh. Geographentag Würzburg, Verhandl. des Deutschen Geographentages, 31: 201-214, 4 Abb.; Wiesbaden (Steiner).

- (1967): Klimaschwankungen in historischer Zeit. Rudloff, H. v. [Edit.]: Die Schwankungen und Pendelungen des Klimas in Europa seit dem Beginn der regelmäßigen Instrumenten-Beobachtungen (1670), 1-370, 89 Abb.; Braunschweig (Vieweg).

- (1985): Das Problem der Klimaänderungen in Vergangenheit und Zukunft. - Erträge der Forschg., 220: 1-228, 35 Abb., 12 Tab.; Darmstadt (Wiss. Buchges.).

GribBin, J. \& LamB, H. H. (1978): Climatic Change in historical times. - GribBin, J. [Edit.]: Climatic Change, 68-82, 4 Fig.; Cambridge (Univ. Press).

HÖppner, H. \& STEeger, A. (1936): Das Naturschutzgebiet „Wisseler Dünen” am unteren Niederrhein. Rhein. Heimatpflege, 1: 92-98, 5 Abb.; Düsseldorf.

Jelgersma, S., DeJong, J., Zagwijn, W. H. \& Van Rfgteren Altena, J. F. (1970): The Coastal Dunes of the Western Netherlands. Geology, Vegetational History and Archeology. - Meded. Rijks Geol. Dienst., N.S., 21: 93-167; Maastricht.

KuIjN, J. A. (1990): The younger Dunes in the Netherlands; Chronology and Causation. - Catena, Suppl. 18: $89-100,3$ Tab., 4 Fig.; Cremlingen.

LAmB, H. H. (1966): The changing Climate, 1-236; London (Methuen).

- (1979): Climatic Variations and Changes in the Wind and Ocean Circulation: The Little Ice Age in the Northeast Atlantic. - Quaternary Research, 11: 1-20, 11 Fig.; New York-London.

- (1982): Reconstruction of the course of postglacial climate over the world. - Harding, A.F. [Edit.]: Climatic Change in Later Prehistory, 11-32, 15 Fig.; Edinburgh (Univ. Press).

Lenke, W. (1968): Das Klima Ende des 16. und Anfang des 17. Jahrhunderts nach Beobachtungen von Tycho de Brake auf Hven, Leonhard III. Treuttwein in Fürstenfeld und David Fabricius in Ostfriesland. Ber. Deutscher Wetterd., 15, 110: 1-49, 3 Abb., 6 Tab.; Offenbach/M.
Leuze, E. (1969): Die Halbinsel von Devon und Cornwall. - Deutsche geogr. Blätter, Geogr. Ges. Bremen, 50: 215-355, 14 Abb., 15 Bild., 5 Kart.; Bremen.

Mückenhausen, E., Scharenseel, H. W. \& Pietig, F. (1968): Zum Alter des Plaggeneschs. - Eiszeitalter u. Gegenwart, 19: 190-196, 1 Abb., 1 Tab.; Öhringen.

PfISTER, Ch. (1982): Klimageschichte der Schweiz 15251860. - Academica helvetica 6, 1-163, 3. Auflage; Bern-Stuttgart (Haupt).

RoBerts, M. J. (1985): The geomorphology and stratigraphy of the Lizard Loess in South Cornwall, England. - Boreas, 14: 75-82, 4 Fig.; Oslo.

SieberTz, H. (1988): Die Decksedimente auf dem Niederrheinischen Höhenzug in ihrer Beziehung zu den Luftdruck- und Windverhältnissen während der Weichsel-Kaltzeit in Nordwestdeutschland. Natur am Niederrhein, 3: 1-12, 8 Abb., 3 Tab.; Krefeld.

- (1994): Chronostratigraphische Untersuchungen (14C-Alter) an den Windablagerungen der „Towans” in der südwestenglischen Grafschaft Cornwall. Eiszeitalter u. Gegenwart, 44: 1-6, 2 Abb., 1 Tab.; Hannover.

Tooley, M. J. (1990): The Chronology of Coastal Dune Development in the Unided Kingdom. - Catena, Suppl. 18: 81- 88, 2 Fig.; Cremlingen.

Weninger, B. (1995): Erläuterungen zu den Ergebnissen der ${ }^{14} \mathrm{C}$-Altersbestimmung; Köln (Inst. Ur- und Frühgeschichte).

Wilson, P. (1990): Coastal Dune Chronology in the North of Ireland. - Catena, Suppl. 18: 71-79, 4 Fig.; Cremlingen.

Manuskript eingegangen am 10. April 1997 